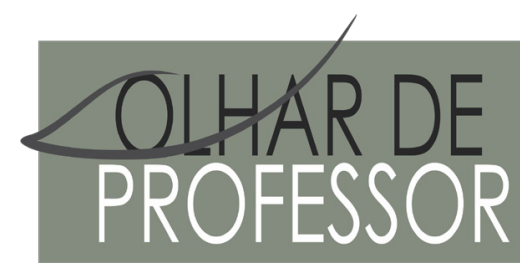

DOI: 10.5212/OLHARPROFR.v.23.2020.15636.209209229631.0720

\title{
POLÍTICAS EDUCACIONAIS DA NOVA GESTÃO PÚBLICA NO PERU: OS DOCENTES NA CULTURA DA PERFORMATIVIDADE EDUCACIONAL
}

\author{
POLICIES OF THE NEW PUBLIC MANAGEMENT IN PERU: TEACHERS IN THE CULTURE OF PERFORMATIVITY \\ POLÍTICAS EDUCATIVAS DE LA NUEVA GESTIÓN PÚBLICA EN PERÚ: LOS DOCENTES EN LA CULTURA DE LA \\ PERFORMATIVIDAD
}

BETHZABÉ COTRADO*

\begin{abstract}
Resumo: Este artigo analisa a construção da cultura da performatividade a partir da configuração das políticas educacionais da Nova Gestão Pública (NGP) no Peru. O estudo, de natureza qualitativa, foi orientado sobre os princípios da teoria fundamentada. Três disposições políticas do NGP, que, atualmente, regulam o ambiente educacional peruano, foram definidas por meio da análise de conteúdo: descentralização e autonomia da gestão educacional, avaliações padronizadas do desempenho da aprendizagem e bônus econômicos. Foi proposta uma entrevista semiestruturada com onze atores educacionais da região de Puno, a fim de conhecer suas experiências pedagógicas e entender seus processos de identidade. Os resultados mostraram traços performativos entre professores e diretores, além de atitudes inseguras em suas práticas pedagógicas. Conclui-se que a incorporação das medidas políticas da NGP promoveria o desenvolvimento de práticas performativas sustentadas na eficácia, na competitividade e na melhoria dos resultados da aprendizagem.
\end{abstract}

Palavras-chave: Professor. Avaliação externa. Performatividade. Política educacional.

\begin{abstract}
This article analyzes the construction of the culture of performance based on the configuration of the educational policies of the New Public Management (NPM) in Peru. The qualitative study was guided by the principles of grounded theory. Through content analysis, three political provisions of the NPM that currently regulate the Peruvian educational sphere have been defined: decentralization and autonomy of educational management, standardized assessments of learning achievement, and economic bonuses. A semi-structured interview was proposed with eleven educational authorities from the Puno region, in order to learn about their pedagogical experiences and understand their identity processes. The results showed performative traits among teachers and managers, as well as insecure attitudes in their pedagogical practices. It is concluded that the incorporation of the NPM's political measures would be promoting the development of performative practices sustained in the effectiveness, competitiveness and improvement of learning outcomes.
\end{abstract}

Keywords: Teacher. Standardized assessments. Performativity. Educational policy.

Resumen: Este artículo analiza la construcción de la cultura de la performatividad a partir de la configuración de las políticas educativas de la Nueva Gestión Pública (NGP) en Perú. El estudio de naturaleza cualitativa se orientó en los principios de la teoría fundamentada. Se ha definido mediante el análisis de contenido tres disposiciones políticas de la NGP que actualmente regulan el ámbito educativo peruano: descentralización y autonomía de gestión educativa, evaluaciones estandarizadas del logro de aprendizaje y bonos económicos. Se planteó una entrevista semiestructurada a once actores educativos de la región Puno, con la finalidad de conocer sus experiencias

\footnotetext{
* Doctora en ciencias de la educación por la Universidad Nacional del Altiplano - Perú. Máster Universitario en Investigación en Educación por la Universidad Autónoma de Barcelona - España; Máster Universitario en Didáctica de la Matemática por la Universidad de Granada - España. Docente de la Universidad Nacional del Altiplano - Perú. E-mail: bethzabe.cotrado@e-campus.uab.cat
} 
pedagógicas y comprender sus procesos de identidad. Los resultados evidenciaron rasgos performativos entre docentes y directivos, así como actitudes inseguras en sus prácticas pedagógicas. Se concluye que, la incorporación de las medidas políticas de la NGP, estaría impulsando al desarrollo de las prácticas performativas sostenidas en la efectividad, competitividad y mejora de resultados de aprendizaje.

Palabras clave: Docente. Evaluación estandarizada. Performatividad. Política educativa.

\section{INTRODUCCIÓN}

El neoliberalismo comprendido como una práctica política, social y cultural se ha convertido en una condición común de los últimos años (ENGLAND; WARD, 2011; PECK; TICKELL, 2002; SPRINGER, 2010). Como un "tsunami económico", ha cobrado fuerzas en todo el planeta, generando procesos de "profundas transformaciones" que afectan a cualquier ámbito de la vida (FAIR, 2008; HARVEY, 2007; ONG, 2007).

En el espacio educativo, la mayoría de los gobernantes de diferentes países, se han centrado en incorporar modelos políticos neoliberales que apuestan por nuevos mecanismos de gestión pública bajo el lema de "reforma educativa” (BALL; YOUDELL, 2008; LUENGO; SAURA, 2012; NARODOWSKI; MARTÍNEZ, 2016). La presencia de las reformas de políticas educativas con fines privatizadores es defendida por un grupo de actores políticos que incluye colectivos de interés locales, organismos internacionales y think tanks privados (BALL; YOUDELL, 2008; SAURA, 2015; SAURA; LUENGO, 2015a; VERGER; NORMAND, 2015).

Una aproximación amplia de las nuevas formas de privatización encubierta en la educación pública es diferenciada por Ball y Youdell (2008) en privatización exógena y privatización endógena. La primera, abre la educación pública a la participación de agentes privados con ánimos de lucro y, la segunda, se vincula con los principios de la Nueva Gestión Pública.

Con el fin de mejorar la eficiencia, eficacia y rendimiento de los sistemas educativos, la adopción de los principios y métodos de la NGP se concretan en una serie de configuraciones políticas siguientes: descentralización de responsabilidades y autonomía de gestión a nivel de instituciones educativas, rendición de cuentas orientados en los resultados de las evaluaciones estandarizadas e incentivos económicos, y evaluación constante de conocimientos didácticos de los docentes (BALL; YOUDELL, 2008; LUENGO; SAURA, 2012; VERGER, et al., 2016).

La incorporación de estas políticas educativas se impone mediante discursos hegemónicos sin reflexión y se aceptan en las normas y reglamentaciones de los sistemas educativos (BERNAL; LORENZO, 2012). Sin embargo, su concreción en las instituciones educativas está originando nuevas formas de entender la educación, nuevas maneras de actuar, nuevos valores y nuevas prácticas de interacción entre directivos, docentes y estudiantes (BALL, 2008). Esto genera de manera significativa el desarrollo de una cultura performativa que remodela las actividades y subjetividades de los sujetos y organizaciones (BALL, 2003 LICINIO; SANTOS, 2004; LUENGO; SAURA, 2013).

En esta perspectiva educativa global, el gobierno peruano ha apostado por los principios y configuraciones políticas de la NGP como una de las reformas más importantes impulsadas desde al año 2000. En el marco de este enfoque, se ha diseñado normas y leyes que han regulado la implementación progresiva de políticas de gestión descentralizadoras y rendición de cuentas bajo la lógica de resultados centrados en el logro de aprendizajes de los estudiantes.

Desde esta realidad educativa actual, se planteó analizar la construcción de la cultura de la performatividad a partir de la configuración de las políticas educativas de la Nueva Gestión Pública en Perú. Para dar respuesta a la problemática se revisó trabajos empíricos sobre las políticas neoliberales de privatización endógena y la cultura de la performatividad (BALL, 2003; BALL; YOUDELL, 2008; JEFFREY; TROMAN, 2011; LICINIO; SANTOS, 2004; LUENGO; SAURA, 2012; LUENGO; SAURA, 2013; MURRAY, 2012). Luego, se identificó tres disposiciones políticas de la NGP que actualmente regulan el ámbito educativo peruano: descentralización de gestión educativa, evaluaciones estandarizadas 
del logro de aprendizaje y bonos económicos. Consecuentemente, se examinaron las entrevistas de los docentes y directivos de la región Puno, con la finalidad de conocer sus experiencias pedagógicas y comprender sus procesos de identidad.

\section{POLÍTICAS EDUCATIVAS DE PRIVATIZACIÓN ENDÓGENA Y LA CULTURA DE LA PERFORMATIVIDAD}

La incorporación de prácticas políticas que están generando cambios contemporáneos en los sistemas educativos de diferentes países, se resaltan como formas encubiertas de privatizar la educación pública (BALL; YOUDELL, 2008). Estas formas encubiertas de privatizar la educación son categorizadas por Ball y Youdell (2008, p. 8) como privatización “endógena” y “exógena”. La privatización endógena o endo-privatización, se refiere a la "importación de ideas, métodos y prácticas del sector privado" a los quehaceres del sistema educativo público. Su concreción se realiza a través de la incorporación de políticas vinculadas con la Nueva Gestión Pública (BALL, 2008). Mientras que la privatización exógena tiene que ver con la "incorporación del sector privado a los establecimientos públicos de enseñanza". Estas prácticas exógenas se concretan en: Asociaciones Público Privados (APP), subvenciones de organismos internacionales, fundaciones filantrópicas y consultorías externas (BALL; YOUDELL, 2008, p. 31).

Las dos formas de privatizar no se excluyen, sino que interactúan mutuamente en la organización y gestión de los sistemas educativos. En esta perspectiva, este estudio enfatiza la NGP como principio organizacional y operacional de los mecanismos de privatización endógena en la educación pública (BALL; YOUDELL, 2008).

La NGP entendida como un enfoque de reforma del sector público que "aplica conocimientos e instrumentos de la gestión empresarial y otras disciplinas” (VIGODA, 2003, p. 813), es el medio principal de la reingeniería cultural de los servicios educativos públicos en los últimos años (BALL; YOUDELL, 2008). Con el fin de mejorar la eficiencia, eficacia y rendimiento de los sistemas educativos, los principios y métodos de la NGP se concretan en una serie de configuraciones políticas siguientes: descentralización de responsabilidades y autonomía de gestión a nivel de instituciones educativas, rendición de cuentas orientados en los resultados de las evaluaciones estandarizadas e incentivos económicos, información pública de resultados por medio de ránquines, evaluación constante de conocimientos didácticos a los docentes y remuneración docente basados en criterios de meritocracia (BALL; YOUDELL, 2008; FALABELLA; DE LA VEGA, 2016; LUENGO; SAURA, 2012; VERGER; NORMAN, 2015; VERGER, et al., 2016).

El establecimiento de estas diversas prácticas políticas de reforma de la educación y modelos de gobernanza de gestión basadas en desempeños, sistemas de rendición de cuentas y responsabilizaciones, están construyendo nuevas formas de control de rendimiento de servicios públicos y nuevas formas de compromisos y prioridades. No son simples mecanismos para un cambio técnico y estructural de las organizaciones, sino dispositivos que se adentran a la identidad de los profesionales del sector público (BALL, 2003).

Las políticas de privatización endógena y las nuevas formas regulativas que desencadenan de los principios de la NGP, apertura condiciones para desarrollar una cultura que remodela las actividades y subjetividades del ser profesional (BALL, 2003). De forma más significativa, el desarrollo de esa cultura se expresa en el uso de la definición de la performatividad de Jean-François Lyotard y Stephen J. Ball. Para Lyotard (1984) la performatividad está centrada en la "optimización de la relación global de sus inputs con sus outputs” (p.12). Esto implica, la maximización de la eficiencia/eficacia del desempeño de un sistema social en función de la economía.

Por su parte Ball (2003), refiriéndose al contexto educativo, define la performatividad como "una tecnología, una cultura y una modalidad de reglamentación que utiliza evaluaciones, comparaciones e indicadores como medios para controlar, desgastar y producir cambio" en los profesionales o instituciones (p. 89 - 90). En un ambiente performativo las relaciones entre sujetos y organizaciones quedan reducidos a indicadores y medidas de competencia, productividad y desempeño acorde a las exigencias del mercado competitivo. 
Es decir, los sujetos son reconocidos como profesionales competentes y disponibles para el mercado de trabajo cuando se caracterizan por estados de desempeño y perfección inalcanzable (BALL, 2003; BALL, 2008; JEFFREY; TROMAN, 2011; LICINIO; SANTOS, 2004). De esta forma la noción de desempeño "significa o representa la valía, la calidad, o el valor de un individuo" que invita y motiva a convertirse en profesionales o instituciones más efectivos dentro de un ámbito evaluativo (BALL, 2003, p. 90). Sin embargo, las medidas y metas de desempeño no se logran en un solo momento, sino durante un proceso de constante competencia y rendición de cuentas, establecido en recompensas y sanciones (BALL, 2010).

De allí pues, la lógica de la cultura de la performatividad competitiva de la NGP trabaja sutilmente para inculcar acciones empresariales en la actitud, en el comportamiento, en el sentido del "yo", en la autovaloración y en el alma de los profesionales a través de: constantes evaluaciones, publicaciones y ránquines de resultados, inspecciones, auditorias, aseguramiento de la calidad, sistema de monitoreos y controles e informes y comparaciones de desempeño (BALL, 2003; BALL, 2008; LUENGO; SAURA, 2013; MOREIRA, 2009). En la aplicación de las tareas basadas en la competición, eficiencia y productividad, los profesionales y organizaciones intentan lograr las metas y objetivos establecidos, por lo que asumen toda la responsabilidad y se comprometen en mejorar los resultados medidos en un afán interminable por la excelencia (BALL, 2003; JEFFREY; TROMAN, 2011; LICINIO; SANTOS, 2004).

Entonces, a medida que estas acciones se desempeñan acorde a las normas y controles disciplinarios que se imponen a las prácticas de trabajo, las satisfacciones laborales y las identidades de los profesionales se van alterando y reconfigurando en términos de costo y beneficio (BALL, 2008). Lo que significa establecer una nueva actitud de orgullo, de asumir responsabilidades de trabajar con mayor dedicación, prestigio académico o triunfo personal por el producto o calidad de los servicios prestados (BALL, 2008; LUENGO; SAURA, 2013). Por otro lado, generan ambivalencia (BAUMAN, 2005), insatisfacción, sentimientos de vergüenza, culpa, inferioridad, inestabilidad y envidia (BALL, 2003; BALL, et al., 2013; LUENGO; SAURA, 2013; MOREIRA, 2009). En ese contexto de desempeño controlado, los efectos perversos de las nuevas formas de poder o tecnologías performativas en el sector público, se ven reflejados en los altos índices de estrés emocional, problemas de salud fisiológica y fracasos relacionados directamente con el trabajo (BALL, 2003; LICINIO; SANTOS, 2004; LUENGO; SAURA, 2013).

\section{METODOLOGÍA}

El estudio se orienta desde el paradigma hermenéutico interpretativo, siguiendo la naturaleza valorativa del enfoque cualitativo y los principios de la teoría fundamentada (STRAUSS; CORBIN, 2002; DENZIN; LINCOLN, 2012). Las estrategias y técnicas utilizadas para recolectar información son: el análisis de contenido cualitativo de los documentos normativos y las entrevistas online en profundidad vía Skype (SULLIVAN, 2012; VALLES, 2007).

Siguiendo la configuración de las políticas educativas orientadas en los principios de la NGP, se han seleccionado documentos legislativos y normativos vigentes y oficiales que orientan las políticas educativas en el ámbito peruano. Con el apoyo de la técnica de análisis de contenido cualitativo (FRAENKEL; WALLEN; HYUN, 2011) de los documentos normativos, se ha establecido tres subcategorías: descentralización de gestión educativa, evaluaciones estandarizadas del logro de aprendizaje y bonos económicos. Estas categorías previas han orientado la ejecución de las entrevistas online en profundidad vía Skype cara a cara y en tiempo real a través del uso de ordenadores y teléfonos inteligentes (SULLIVAN, 2012).

Para el proceso de la entrevista, la población se ha delimitado en los docentes y directivos de nivel primaria y secundaria de la región Puno. La selección de los participantes fue realizada mediante el muestreo en cadena o bola de nieve considerando los siguientes criterios de selección: (a) predisposición de participación y colaboración en la investigación, (b) conocimientos básicos y manejo de las tecnologías de información y comunicación, (c) condición laboral y (d) tiempo de servicios (más de cinco años) en instituciones educativas públicas. De este proceso se eligieron once (11) informantes potenciales.

Con expectativas de formalizar los procedimientos éticos de investigación, antes de pactar la entrevista online, se envió a los correos electrónicos personales la carta de consentimiento informado 
explicando brevemente sobre los objetivos de la investigación. A fin de mantener confidencialidad y anonimato de la información proporcionada de cada participante (MESIA, 2007), se les asigna pseudónimos y unas iniciales que identifican: docente de primaria (DP), docente de secundaria (DS) y directivo (D).

\section{CONFIGURACIONES POĹTIICAS DE LA NGP EN EL SISTEMA EDUCATIVO PERUANO}

En los últimos años el enfoque de la NGP ha penetrado con fuerza a las agendas educativas de países industrializados, así como en vías de desarrollo (VERGER; NORMAND, 2015). Cada territorio de acuerdo a sus características institucionales, político-administrativas y culturales ha adoptado y promulgado los principios, medidas y lógicas de la NGP de diversas formas. En algunos países se ha recontextualizado en políticas de mercado y privatización mientras que, en otros en procesos de descentralización y rendición de cuentas (VERGER; NORMAND, 2015).

En el ámbito educativo peruano la adopción de la NGP se ha concretado en procesos de descentralización de gestión educativa y rendición de cuentas sobre los resultados de logros de aprendizaje. En esa perspectiva se han identificado tres prácticas políticas incorporadas durante procesos de reforma educativa de los últimos tiempos: descentralización de responsabilidades de gestión educativa, rendición de cuentas basadas en evaluaciones estandarizadas y bonos económicos como incentivo docente.

\section{AJ DESCENTRALIZACIÓN DE RESPONSABILIDADES Y AUTONOMÍA DE GESTIÓN A NIVEL DE INSTITUCIONES EDUCATIVAS}

En las últimas décadas el proceso de descentralización se ha concretado a través de la distribución de poder y fragmentación de instancias de gestión educativa. Según la Ley General de Educación 28044, las instancias de gestión son: la Institución Educativa, la Unidad de Gestión Educativa Local (UGEL), la Dirección Regional de Educación (DRE) y el Ministerio de Educación (MINEDU). A cada una de las instancias de gestión se han transferido funciones, responsabilidades y competencias específicas compartidas, de tal manera que gozan de autonomía pedagógica y de gestión de acuerdo con las normas vigentes (ÁLVAREZ, 2010).

Así, el nuevo organigrama del sistema educativo encabeza el MINEDU, como órgano del Gobierno Nacional que gestiona políticas nacionales en corresponsabilidad con las regiones. A nivel de Gobierno Regional las DRE definen políticas regionales y coordinan con las UGEL el funcionamiento de las instituciones educativas. A nivel local, las UGEL concentran cuestiones administrativas y brindan asistencia técnica a las instituciones educativas para lograr su autonomía. Por último, las instituciones educativas son la primera y principal instancia de gestión del sistema educativo descentralizado. En ellas, los poderes de decisión se distribuyen en cuanto al manejo presupuestario de la institución educativa y la autonomía de la conducción pedagógica (CONSEJO NACIONAL DE EDUCACIÓN, 2007).

En el contexto de autonomía pedagógica, una de las estrategias que se propone en la política de modernización y descentralización de la gestión educativa es "Fortalecer la institucionalidad de la institución educativa, a través de una gestión educativa descentralizada, participativa, transparente que brinde un servicio de calidad centrada en el logro de aprendizaje” (MINEDU, 2013, p. 58). En el proceso de un servicio de calidad y logros de aprendizaje las medidas que mayor fuerza toman son las políticas de autonomía escolar. La autonomía escolar orienta las funciones del director en la perspectiva de una gestión basada en liderazgo pedagógico y rendición de cuentas referidos al logro de aprendizajes.

\section{B] RENDICIÓN DE CUENTAS BASADAS EN EVALUACIONES ESTANDARIZADAS DEL LOGRO DE APRENDIZAJE DE LOS ESTUDIANTES}

En la actualidad el desarrollo de las "evaluaciones estandarizadas como forma de conocer mejor la dinámica de procesos y resultados en los sistemas educativos" es una tendencia creciente a nivel nacional e internacional (RAVELA, et al., 2008, p. 46). A nivel internacional, el instrumento biopolítico de mayor 
control de sistemas educativos de varios países es el Program for International Student Assessment (PISA) de la Organización para la Cooperación y el Desarrollo Económico (OCDE).

A nivel nacional, como es el caso peruano el sistema de evaluaciones de aprendizajes estandarizados es conocido como Evaluación Censal de Estudiantes (ECE) (CUETO, 2007; PÉREZ, 2015). Esta prueba estandarizada de gran escala se ampara en la Ley General de Educación y el Proyecto Nacional de Educación. Asimismo, su fundamentación se complementa con la promulgación de decretos (D.S. $\mathrm{N}^{\circ}$ 021-2007-ED; D.S. $\mathrm{N}^{\circ}$ 015-2008-ED, D.S. No 017-2015-MINEDU) que respaldan su permanente implementación, ejecución y difusión de resultados. Su cumplimiento y publicación de resultados de los logros de aprendizaje se desarrolla anualmente en coordinación de la Oficina de Medición de la Calidad de los Aprendizaje (UMC) y el Ministerio de Educación.

\section{C] BONOS ECONÓMICOS COMO INCENTIVOS AL DESEMPEÑO ESCOLAR}

Esta medida del mundo empresarial (CASSETTARI, 2008) utilizada para incentivar a los docentes y directivos por su buen desempeño, entra en vigencia a partir del 2014 en la educación peruana. Mediante el Decreto de Urgencia $N^{\circ}$ 002-2014 se autoriza al Ministerio de Educación y a los gobiernos regionales otorgar el "Bono de incentivo al desempeño escolar como reconocimiento de la mejora en el aprendizaje de los estudiantes de las instituciones educativas públicas" a favor de los profesores y directivos con mejores desempeños en la prueba nacional estandarizada (ECE).

\section{DOCENTES Y DIRECTIVOS EN LA CONSTRUCCIÓN DE LA CULTURA DE LA PERFORMATIVIDAD}

En el apartado anterior se ha identificado tres prácticas políticas de la NGP que regulan actualmente el sistema educativo público peruano. En ese contexto, se analizan y exponen las diferentes narrativas de las prácticas y subjetividades de los actores educativos que han participado en esta investigación. El análisis de las narrativas se ha concretado en dos subcategorías: prácticas performativas entre docentes y directivos y reconfiguración de la identidad docente.

Estas categorías dan a entender que en el espacio educativo los agentes educativos están asumiendo nuevos roles y prácticas performativas: competitivas, comparativas, incentivaciones económicas basadas en desempeños, intensificación de trabajo, control y monitoreos constantes (BALL, 2003; HARGREAVES, 2005; SAURA; LUENGO, 2015b). La suma de estos actos y medidas de la performatividad están produciendo y generando cambios severos en la interacción social y la identidad de los docentes (BALL, 2003; BALL, 2010; JEFFREY; TROMAN, 2011; LUENGO; SAURA, 2013; MURRAY, 2012).

\section{AJ PRÁCTICAS PERFORMATIVAS ENTRE DOCENTES Y DIRECTIVOS}

A lo largo de las décadas, la competencia se ha extendido a todas las organizaciones sociales llegando a ser "una de las fuerzas más poderosas de la sociedad para avanzar en muchos ámbitos del esfuerzo humano" (PORTER, 2009). Su introducción al espacio de las políticas públicas llega de la mano con las ideas y técnicas propias de las empresas "bajo el imperativo de competitividad y rentabilidad" (RODRÍGUEZ; DÍEZ, 2014, p. 385).

En el ámbito educativo la competitividad se estaría instaurando como una nueva cultura "empresarial competitiva” caracterizada por proceso de evaluación, comparación, logro de metas e incentivos por desempeño (BALL, 2003; SAURA; LUENGO, 2015b). En este ambiente donde legitima términos de eficacia, productividad y optimización de resultados (BALL, 2003). Las instituciones educativas, docentes y estudiantes asumen el rol de competir entre sí, para que los resultados de las mediciones estandarizadas proporcionen a toda la comunidad educativa información cuantitativa detallada y clasificada en tablas de posicionamiento y gráficos estadísticos que faciliten la comparación entre las "unidades mínimas de medición” (SAURA; LUENGO, 2015b, p. 127). 
La base de datos, la publicación de información en rankings sobre resultados académicos y las comparaciones sirven como tácticas para visibilizar el mejor o peor desempeño organizacional de las instituciones educativas (PERRY; MCWILLIAM, 2009). Estas medidas interrelacionadas en un sistema de accountability performativa, movilizan las acciones de los actores al logro de estándares y resultados de aprendizaje prefijados desde el estado (BALL, 2003; DARLING-HAMMOND, 2004; FALABELLA; DE LA VEGA, 2016). Su empleo tiene diferentes consecuencias: incremento de competitividad entre actores educativos por una posición en los rankings, comparaciones entre instituciones educativas, intensificación de trabajo, control y monitoreos constantes. Estas acciones se corroboran con la narración de la experiencia de los docentes y directivos:

(...) estos días de la semana tenemos que hacer fructíferos, en el sentido de que tenemos que integrarnos para revertir los resultados de la evaluación, no es posible que las instituciones educativas rurales con la peor infraestructura y menor cantidad de estudiantes estén mejor que nosotros (Fátima - DS).

En esta narrativa la profesora pone de manifiesto que la competitividad ha sido instalada en su institución educativa. Frente a los resultados poco favorables de la ECE, los actores educativos asumen plena responsabilidad para focalizar su desempeño en tareas evaluables. Esta herramienta de control enfocado en el cuerpo docente orienta a que los desempeños se ajusten en intentos de emulación constante y comparaciones entre instituciones educativas (CASASSUS, 2007; BALL, 2010; SAURA; LUENGO, 2015b). Este hecho origina "seres dividuales", es decir seres dependientes unos de otros, que se necesitan entre sí, para producir resultados al máximo cociente (SAURA; LUENGO, 2015b, p. 130). Así lo demuestra esta profesora que tiene adentrado su dividualidad:

En mi IE los que hemos estado con segundo grado nos hemos apoyado entre nosotros, sacrificamos muchas horas de trabajo para la evaluación ECE, obtuvimos buenos resultados, pero no todos recibimos el bono escuela (Bertha - DP).

En un ambiente competitivo los profesores como sujetos dependientes entre sí, asumen plenamente comportamientos de esfuerzo y superación orientados al logro de metas altas establecidos mediante estándares. Estas medidas de estandarización pueden generar efectos perversos, así como: trabajar en función de beneficios económicos, dejar de lado la reflexión por la formación individual e integral del alumno o del aula, tácticas tramposas que faltan a la ética (excluir de las evaluaciones a estudiantes con atraso académico) o entrenamiento académico repetitivo en las áreas evaluables (SAURA; LUENGO, 2015b; FALABELLA; DE LA VEGA, 2016). Así da a conocer este director sobre las decisiones adoptadas en su institución respecto a la ECE:

(...) yo mismo cometí el error el año pasado de hacer que una semana antes de la prueba nos hemos dedicado a hacer solo matemática y comunicación y ni aun eso hemos podido lograr las metas propuestas (...) (Gonzalo - D).

El relato del director demuestra que la instrucción repetitiva fue tomada como una opción para lograr metas en las áreas evaluables de las pruebas estandarizadas. En este tipo de prácticas de rendimiento, la formación integral del estudiante desvanece y el acto de enseñar del profesor son alterados (BALL, 2003). Estas prácticas performativas tecnifican, desprofesionalizan y somete la praxis educativa del docente a una reorganización de entrenamiento y creciente intensificación de trabajo (FALABELLA; DE LA VEGA, 2016; HARGREAVES, 2005), así como señala este director:

(...) ahora los profesores están dando más tiempo y eso también hacen las demás IE quienes han logrado mejores resultados, los profesores trabajan horas extras e incluso algunos profesores han obligado a los padres de familia que contraten profesores particulares, mira a ese extremo estamos llegando (Kevin - D).

Los directivos escolares en afán de lograr resultados de rendimiento académico positivos e información de la práctica pedagógica, utilizan mecanismos estratégicos para cambiar la actitud del 
profesor a través de monitoreo permanente. Movilizan todo tipo de recursos para que la organización funcione bien y apoyan la intensifican del trabajo docente. Las políticas de medición estandarizada, rendición de cuentas e incentivos económicos, son dispositivos de explotación de fuerza de trabajo docente más sutiles e invisibles en el interior de las instituciones educativas. Los profesores para responder a las exigencias de estas políticas incorporan en su trabajo nuevas actividades y responsabilidades, que en muchos casos son tomados como innovadoras e indispensables (ANDRADE; FELDFEBER, 2006). Como este docente señala su experiencia:

(...) me llevo todo el material de trabajo necesario a mi aula para enseñar comunicación y matemática a mis estudiantes, no me importa si pierdo el almuerzo, me quedo treinta minutos más de la hora de salida con ellos, lo que me importa es que todos aprendan y saquen buenas notas en las evaluaciones programadas (Lukas - DP).

El profesor, a pesar de la carga de trabajo, pone en marcha las actividades para aligerar esta sobrecarga de trabajo impuesto, sacrificando su salud y afectando su identidad. Las reformas educativas actuales mediante pautas de "vigilancia administrativa y de control burocrático expresan la persistencia de la misión modernista” en los sistemas educativos (HARGREAVES, 2005, p. 138). Impulsadas por las prácticas de la NGP, ejercen y orienta un mayor control y vigilancia del tiempo y del trabajo docente. Las políticas de medición estandarizada y los incentivos económicos por desempeño son las medidas actuales más impactantes que originan profundas alteraciones en la organización de los centros escolares (BARBOSA; FERNANDES, 2013).

En este sentido, el comportamiento de los sujetos es guiado mediante esquemas de diferenciación salarial y simbólica, de tal manera que las decisiones maximicen el logro de los resultados de las evaluaciones estandarizadas (LAVAL; DARDOT, 2013). Así lo señala este director orgulloso de haber logrado los buenos resultados en la evaluación ECE y ser beneficiario del bono escuela: "en el año 2013, alcanzamos buenos resultados y hemos recibido el bono" (Kevin - D). Como fenómeno nuevo, los incentivos económicos en las instituciones educativas movilizan, motivan y obligan a corresponder a los actores educativos a las nuevas orientaciones e imperativos de la competencia. Así la incentivación económica cumple su objetivo de reestructurar la praxis pedagógica del docente y trabajar en función a los beneficios económicos. En tal sentido los docentes se comprometen al trabajo en equipo y orientan su conducta a competir con otras instituciones educativas, con fines de lograr los incentivos financieros.

\section{B] RECONFIGURACIÓN DE LA IDENTIDAD DOCENTE}

Los rankings, la competencia, las comparaciones, evaluaciones, la accountability, la intensificación de trabajo, las incentivaciones económicas y la privatización son nuevos mecanismos de poder y control que se han implementado en las instituciones educativas públicas. Estas técnicas disciplinarias de gobernanza crean nuevas vías de reflexión y permite describir acerca de lo que hacen los docentes, de sus valores, de sus roles desde una política de rentabilidad, fundamentados en intereses institucionales privadas y valor performativo (BALL, 2003; BALL; YOUDELL, 2008).

En este escenario, la identidad del profesor está siendo forzada a sucesivas transformaciones sociales, producto de continuas reformas neoliberales educativas, lo cual contribuye a desestabilizar la identidad profesional en vez de mejorar la calidad educativa (BOLÍVAR, 2005). Este docente relata su experiencia de vivir en las reformas educativas, aludiendo que las duras exigencias externas reprimen su razón de ser docente. Frente a ello se siente culpable y que su labor de docencia no tiene sentido.

Cuando yo empecé a trabajar la educación era un poquito flexible, ahora en los últimos años ha sufrido fuertes cambios, hay mucha exigencia con nosotros y nuestros niños, frente a esa situación los docentes antiguos nos sentimos muy golpeados (...) ya no disfruto mi trabajo con mis alumnos, eso me hace sentir mal, (Abel - DP).

El profesor experimenta una contradicción entre lo que piensa y lo que hace, vive con una conciencia bifurcada y en sus propios juicios se crea una ruptura potencial sobre lo que es las necesidades de los 
estudiantes y una buena práctica y las exigencias del desempeño (BALL, 2003). Estos docentes que tratan de manejar contradicciones entre creencias y expectativas, o luchan con emociones reprimidas son "manifestaciones de la ambivalencia que provocan tribulación y sufrimiento" (BAUMAN, 2005, p.12). La agenda educativa actual se fundamenta en que los docentes deben ser presionados en mostrar y alcanzar un set de resultados prescritos externamente. Por consiguiente, los profesores sienten haber perdido la posibilidad de exigir respeto, porque lo único que cuenta es su desempeño, mas no se le permite expresar su opinión, sus inquietudes y sus puntos de vista, solo prima la "razón instrumental” (BALL, 2003), así se desvanece la identidad del docente. Ya no interesan las situaciones personales, lo que si compromete es su contribución a los resultados que exige el sistema mercantilista competitivo.

(...) me siento un poquito satisfecho, pero todavía creo que me falta mucho por mejorar (...) obviamente no lo veo óptimo, ni perfecto, (Daniel - DS).

Aquí el profesor, evidencia sentimientos de inseguridad respecto a su propia praxis educativa, dudas si está haciendo bien o mal su trabajo y experimenta una suma de confusión llamada "ambivalencia" (BAUMAN, 2005). En esta medida el profesor se convierte en un ser "ontológicamente inseguro" (BALL, 2003), en el sentido de si está haciendo lo correcto o incorrecto, tanto como otros o tan bien como ellos, luchando siempre por ser el óptimo, el mejor o el excelente, en afán de ser perfecto.

(...) me estresa, no cumplir y alcanzar las metas, en mi quehacer diario parece que ya se hace normal, sin embargo, siento la preocupación de dar algunos temas más a mis estudiantes (Eduardo - DS).

En el discurso del profesor Eduardo se percibe rasgos de performatividad, preocupación y culpa por no cumplir las tareas y las metas prefijadas. Los profesores se autoevalúan, se culpan por aquello que les fue impuesto hacer y no consiguen realizar. Los constantes controles y prácticas impuestas en la actividad docente desencadenan aumento de presión psicológica, altos índices de estrés y mayores problemas de salud. El agotamiento emocional como dimensión de "síndrome de Burnout y desgaste profesional" (ÁLVAREZ; FERNÁNDEZ, 1991), constituye un elemento estresor que causa deterioro psicológico y fisiológico en los docentes. Este diagnóstico modifica la personalidad del docente, las relaciones sociales y los procesos que conducen a la buena formación integral de los estudiantes (LUENGO; SAURA, 2013).

\section{CONCLUSIONES}

En este estudio se ha identificado la configuración de las políticas educativas vinculadas a la NGP que actualmente regulan y orientan el sistema educativo público peruano: gestión educativa descentralizada, rendición de cuentas basadas en evaluaciones estandarizadas del logro de aprendizaje y bonos económicos como incentivo al desempeño escolar. La puesta en conjunto de estas políticas está generando prácticas y actitudes performativas en el interior de las instituciones educativas y sobre todo en los quehaceres e interacciones sociales de los docentes y directivos.

Las prácticas performativas que se expresan en el aumento de competencia y competitividad entre sujetos e instituciones, comparaciones mediante datos cuantitativos o valores extrínsecos establecidos en tablas y rankings que conllevan a la clasificación y jerarquía de instituciones, monitoreo, vigilancia y control constante a los profesores de parte de los directivos, están generando aumento de actividades e intensificación de trabajo. Junto a estas regulaciones las identidades de los docentes están sufriendo múltiples modificaciones emocionales, así como pérdida de valores, deterioro psicológico y fisiológico, incertidumbre y ambivalencia, miedo, culpa, inestabilidad, resentimientos, afán de ser excelente y orgullo.

Todas estas modificaciones en la praxis educativa de los profesores, así como en sus procesos identitarios, tienen que ver con la cultura de la performatividad (BALL, 2003; BALL, 2010; LUENGO; SAURA, 2013). Estas actitudes en su conjunto son adentradas en la subjetividad del sentido del yo y modifica lo que es ser un profesor. Estimula emociones contradictorias, un yo disyuntivo que reprime y genera estrés, malestar y deterioro fisiológico. A pesar de todas estas prácticas educativas y cambios 
en la identidad, los profesores intentan cumplir las exigencias de las competencias, el logro de metas y objetivos (BALL, 2003).

Para mediar la implementación de estas nuevas prácticas de control externo de los docentes, recaen nuevos roles de mayor responsabilidad en la figura de la dirección escolar. Estos cambios en la dirección tienen que ver con lo que ha sido comprendido como autonomía escolar de gestión (VERGER; NORMAND, 2015). Los directivos en su rol de líder pedagógico y superioridad están creando condiciones para facilitar las prioridades y exigencias de las reformas. La composición de todas estas acciones de dinámicas endógenas de privatización de la educación se instituye en el alma de los profesores y trabaja de afuera hacia adentro y de adentro hacia afuera (BALL, 2010), lo que conlleva a la construcción de la cultura de la performatividad en el interior de las instituciones educativas públicas.

\section{REFERENCIAS}

ÁLVAREZ, N. La descentralización educativa en el Perú. Educación, v. 19, n. 37, p. 7-26, 2010.

ÁLVAREZ, E.; FERNÁNDEZ, L. El Síndrome de” Burnout” o el desgaste profesional. Revista de la Asociación Española de Neuropsiquiatría, v. 11, n. 39, p. 257-265, 1991.

ANDRADE, A; FELDFEBER, M. Políticas educativas y trabajo docente: nuevas regulaciones ¿nuevos sujetos? Buenos aires: Novedades educativas, 2006.

BALL, S.J. Profesionalismo, gerencialismo y performatividad. Educación y Pedagogía, v. 15, n. 37, p. 87-104, 2003.

BALL, S. El desempeño, la privatización, los profesionales de la educación y el estado. Educación y Ciudad, n. 14, p. 115-137, 2008.

BALL S. J. Performatividad y fabricaciones en la economía educacional: Rumbo a una sociedad performativa. Pedagogía y Saberes, n. 38, p. 103-113, 2010.

BALL, S. J.; YOUDELL, D. Privatización encubierta en la educación. Bruselas: Education International, 2008.

BARBOSA, A.; FERNANDES, M. J. D. S. O pagamento por desempenho no contexto das reformas educacionais paulistas. Revista Comunicações, p. 45-59, 2013.

BAUMAN, Z. Modernidad y ambivalencia (Vol. 44). Barcelona: Anthropos Editorial, 2005.

BERNAL, J.L.; LORENZO, J. La privatización de la educación pública. Una tendencia en España. Un camino encubierto hacia la desigualdad. Profesorado. Revista de Currículum y Formación del Profesorado, v. 16, n. 3, p. 81-109, 2012.

BOLÍVAR, A. ¿Dónde situar los esfuerzos de mejora?: Política educativa, escuela y aula. Educação e Sociedade, v. 26, n. 92, p. 859-888, 2005.

CASASSUS, J. El precio de la evaluación estandarizada: la pérdida de calidad y la segmentación social. Revista Brasileira de Política e Administração da Educação-Periódico Científico editado pela ANPAE, v. 23, n. 1, p. 71-79, 2007.

CASSETTARI, N. Pagamento por performance na educação básica. Reunião Anual da ANPED, v. 31, p. 41-42, 2008.

CONSEJO NACIONAL DE EDUCACIÓN. Evaluaciones estandarizadas del rendimiento escolar. Boletín del Consejo Nacional de Educación (36). Lima, Perú, 2013.

CONSEJO NACIONAL DE EDUCACIÓN. Proyecto Educativo Nacional al 2021: La educación que queremos para el Perú. Lima, Perú, 2007. 
CUETO, S. Las evaluaciones nacionales e internacionales de rendimiento escolar en el Perú: Balance y perspectivas. En Grupo de Análisis para el Desarrollo (Ed.), Investigación, Políticas y Desarrollo en el Perú, p. 405-455, 2007.

DARLING-HAMMOND, L. (2004). Standards, Accountability and School Reform. Teachers College Record, v. 106, n. 6, p. 1047-1085, 2004.

DECRETO DE URGENCIA N ${ }^{\circ}$ 002-2014. Dictan medidas extraordinarias y urgentes adicionales en materia económica y financiera para estimular la economía nacional. Consultada en mayo del 2016 en: http://busquedas.elperuano.com.pe/download/url/dictan-medidas-extraordinarias-y-urgentesadicionales-en-mat-du-n-002-2014-1116550-1

DENZIN, N. K.; LINCOLN, Y. S. Manual de investigación cualitativa. Vol. I, El campo de la investigación cualitativa. Barcelona: Gedisa, 2012.

ENGLAND, K.; WARD, K. Neoliberalization: States, networks, peoples (Vol. 30). Oxford: Blackwell, 2011.

FAIR, H. El sistema global neoliberal. Polis (Santiago), v. 7, n. 21, p. 229-263, 2008.

FALABELLA, A.; DE LA VEGA, F. Políticas de responsabilización por desempeño escolar: un debate a partir de la literatura internacional y el caso chileno. Revista Estudios Pedagógicos, v. 42, n. 2, p. 395-413, 2016.

FRAENKEL, J. R.; WALLEN, N. E.; HYUN, H. H. How to design and evaluate research in education. New York: McGraw-Hill Humanities/Social Sciences/Languages, 2011.

HARVEY, D. Breve historia del neoliberalismo. Madrid: Akal, 2007.

HARGREAVES, A. Profesorado, cultura y postmodernidad: cambian los tiempos, cambia el profesorado. Madrid: Morata, 2005.

JEFFREY, B.; TROMAN, G. The construction of performative identities. European Educational Research Journal, v. 10, n. 4, p. 484-501, 2011.

LAVAL, C.; DARDOT, P. La nueva razón del mundo. Barcelona: Gedisa, 2013.

LEY N 28044. Ley General de Educación. Lima Perú. Consultada en marzo del 2016 en: http:// www.minedu.gob.pe/p/ley_general_de_educacion_28044.pdf, 2003.

LICINIO, L.; SANTOS, C. P. Formação de professores na cultura do desempenho. Educação \& Sociedade, v. 25, n. 89, p. 1145-1157, 2004.

LUENGO, J.; SAURA, G. Mecanismos endógenos de privatización encubierta en la escuela pública. Políticas educativas de gestión de resultados y rendición de cuentas en Andalucía. Profesorado.

Revista de Currículum y Formación del Profesorado, v. 16, n. 3, p. 111-126, 2012.

LUENGO, J.; SAURA, G. Dinámicas endógenas de privatización en la educación. La cultura de la performatividad. Revista Iberoamericana sobre Calidad, Eficacia y Cambio en Educación, v. 11, n. 3, p. 139-153, 2013.

LYOTARD, J. F. La condición postmoderna: informe sobre el saber. Madrid: Cátedra, 1984

MESIA, R. Contexto ético de la investigación social. Investigación Educativa, v. 11, n. 19, p. 137 151, 2007.

MINEDU. Política de modernización y descentralización de la gestión en el sector educación. Gestión educativa moderna y descentralizada. Lima, 2013. 
MOREIRA, A. F. A cultura da performatividade e a avaliação da pós-graduação em educação no Brasil. Educação em Revista, v. 25, n. 3, p. 23-42, 2009.

MURRAY, J. Performativity cultures and their effects on teacher educators' work. Research in Teacher Education, v. 2, n. 2, p. 19-23, 2012.

NARODOWSKI, M.; MARTINEZ, A ¿Por qué se expande la educación privada?: Aportes para el debate global. Revista Colombiana de Educación, n. 70, p. 17-26, 2016.

ONG, A. Neoliberalism as a mobile technology. Transactions of the Institute of British Geographers, v. 32, n. 1, p. 3-8, 2007.

PECK, J.; TICKELL, A. Neoliberalizing space. Antipode, v. 34, n. 3, p. 380-404, 2002.

PÉREZ, H. H. Marco de fundamentación de las pruebas de rendimiento de la Evaluación Censal de Estudiantes de $2^{\circ}$ grado de Secundaria 2015. Lima Perú, 2015.

PERRY, L. A.; MCWILLIAM, E. Accountability, responsibility and school leadership. The Journal of Educational Enquiry, v. 7, n. 1, p. 32-43, 2009.

PORTER, M. Ser competitivo. Barcelona: Deusto, 2009.

RAVELA, P.; ARREGUI, P.; VALVERDE, G.; WOLFE, R.; FERRER, G.; MARTÍNEZ, F.; AYLWIN, M; WOLF, L. Las evaluaciones que América Latina necesita. Revista Iberoamericana de Evaluación Educativa, v. 1, n. 2, p. 51 - 63, 2008.

RESOLUCIÓN MINISTERIAL $N^{\circ}$ 0369-2012-ED. Prioridades de la política educativa nacional 2012 - 2016. Lima 26 de septiembre 2012. Consultada en enero del 2016 en: http://busquedas. elperuano.com.pe/normaslegales/aprueban-prioridades-de-la-politica-educativa-nacional-2012resolucion-ministerial-n-0369-2012-ed-846939-3/

RESOLUCIÓN MINISTERIAL Nº 0518-2012-ED. Plan Estratégico Sectorial Multianual de Educación 2012-2016. Lima 19 de diciembre 2012. Consultada en enero del 2016 en: http://www. minedu.gob.pe/normatividad/resoluciones.php

RESOLUCIÓN MINISTERIAL N 0554-2013-ED. Norma para la implementación y ejecución a nivel nacional de evaluaciones del logro de aprendizaje de los estudiantes de educación básica. Lima 08 de noviembre del 2013. http://umc.minedu.gob.pe/wp-content/uploads/2016/04/RM-05542013_NT_evaluaciones.pdf

RODRÍGUEZ, C.; DÍEZ, E. J. Conocimiento y competencias básicas en la formación inicial de maestras y maestros. Profesorado. Revista de Currículum y Formación de Profesorado, v. 18, n. 1, p. 383-396, 2014.

SAURA, G. Think Tanks y educación. Neoliberalismo de FAES en la LOMCE. Archivos Analíticos de Políticas Educativas, v. 23, n. 107, p. 1- 19, 2015.

SAURA, G.; LUENGO, J. J. Política global más allá de lo nacional. Reforma educativa (LOMCE) y el régimen de estandarización (OECD). Bordón. Revista de pedagogía, v. 67, n. 1, p. 135-148, 2015a

SAURA, G.; LUENGO, J. L. Biopolítica y educación. Medición, estandarización, regularización poblacional. Teoría de la Educación; Revista Interuniversitaria, v. 27, n. 2, p. 115-135, 2015b

SPRINGER, S. Neoliberalism and geography: Expansions, variegations, formations. Geography Compass, v. 4, n. 8, p. 1025-1038, 2010.

STRAUSS, A. L.; CORBIN, J. Bases de la investigación cualitativa: técnicas y procedimientos para desarrollar la teoría fundamentada. Medellín: Universidad de Antioquia, 2002 
SULLIVAN, J. R. Skype: An appropriate method of data collection for qualitative interviews. The Hilltop Review, v. 6, n. 1, p. 54-60, 2012.

VALLES, M. S. Entrevistas cualitativas (Vol. 32). Madrid: Centro de Investigaciones Sociológicas, 2007.

VERGER, A.; NORMAND, R. Nueva gestión pública y educación: elementos teóricos y conceptuales para el estudio de un modelo de reforma educativa global. Educação \& Sociedade, v. 36, n. 132, p. 599-622, 2015.

VERGER, A.; ZANCAJO, A.; FONTDEVILA, C. La economía política de la privatización educativa: políticas, tendencias y trayectorias desde una perspectiva comparada. Revista Colombiana de Educación, n. 70, p. 47-78, 2016.

VIGODA, E. New public management. In J. Rabi, Encyclopedia of public administration and public policy, v.2. New York: Marcel Dekker, 2003. 